\title{
Density Spectral Array of BIS VISTA Monitoring System in a functional hemispherectomy
}

\author{
C. Luis, E. Vilà, L. Moltó, J. Fernández Cadil, A. Pardo, S. Pacreu
}

Department of Anaesthesia and Critical Care, Hospital del Mar, Parc de Salut Mar, Barcelona, Spain.

\section{Introduction}

The Bilateral Bispectral index (BIS) was designed to display four channels of EEG; two from each side of the brain. It is useful to detect changes in EEG, and in the power spectrum distribution using the density spectral array (DSA) in both hemispheres. Functional hemispherectomy $(\mathrm{FH})$ is a surgical technique to treat drug-resistant epilepsy caused by extensive hemispheric lesions, usually in children. We present the case of an adult patient scheduled for a right FH where the DSA was helpful for monitoring the effectiveness of the surgery.

\section{Case report}

A 40-year-old woman with extensive inflammation of the right cerebral hemisphere caused by cortical focal dystrophy suffred epileptic crisis and daily disconnections. A section of the mesial frontal lobe had already been made (fig. 1). She was scheduled for a right $\mathrm{FH}$.

The Bilateral BIS electrode strip was placed on the front temporal position. At the beginning of the surgery, we observed a power increase in low frequency $(0.1-4 \mathrm{~Hz})$ and alpha bands (8-12 Hz) in the right hemisphere, where the epileptogenic focus was located (fig.2). After the frontal disconnection, there was a marked decrease of power in low frequency and alpha bands in the right side (fig.3). Subsequently, with temporal, parietal, occipital, insula and corpus callosum disconnection there were no changes in the spectrogram. Unlike DSA, the BIS trend did not reflect differences between two hemispheres. There were not any differences in neurological examination between preoperative and postoperative.

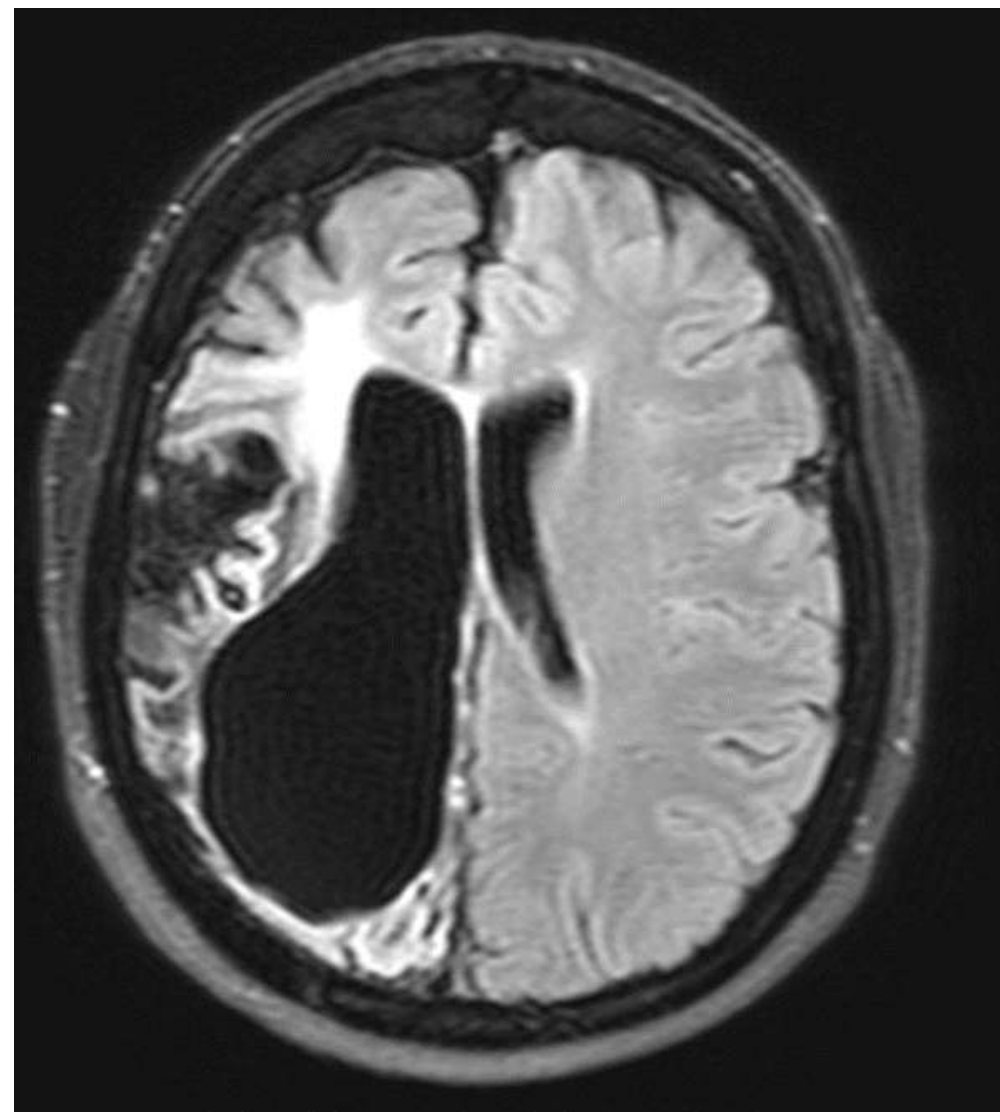

Fig 1. Patient's RMN with cortical focal dystrophy and section of the mesial frontal lobe.

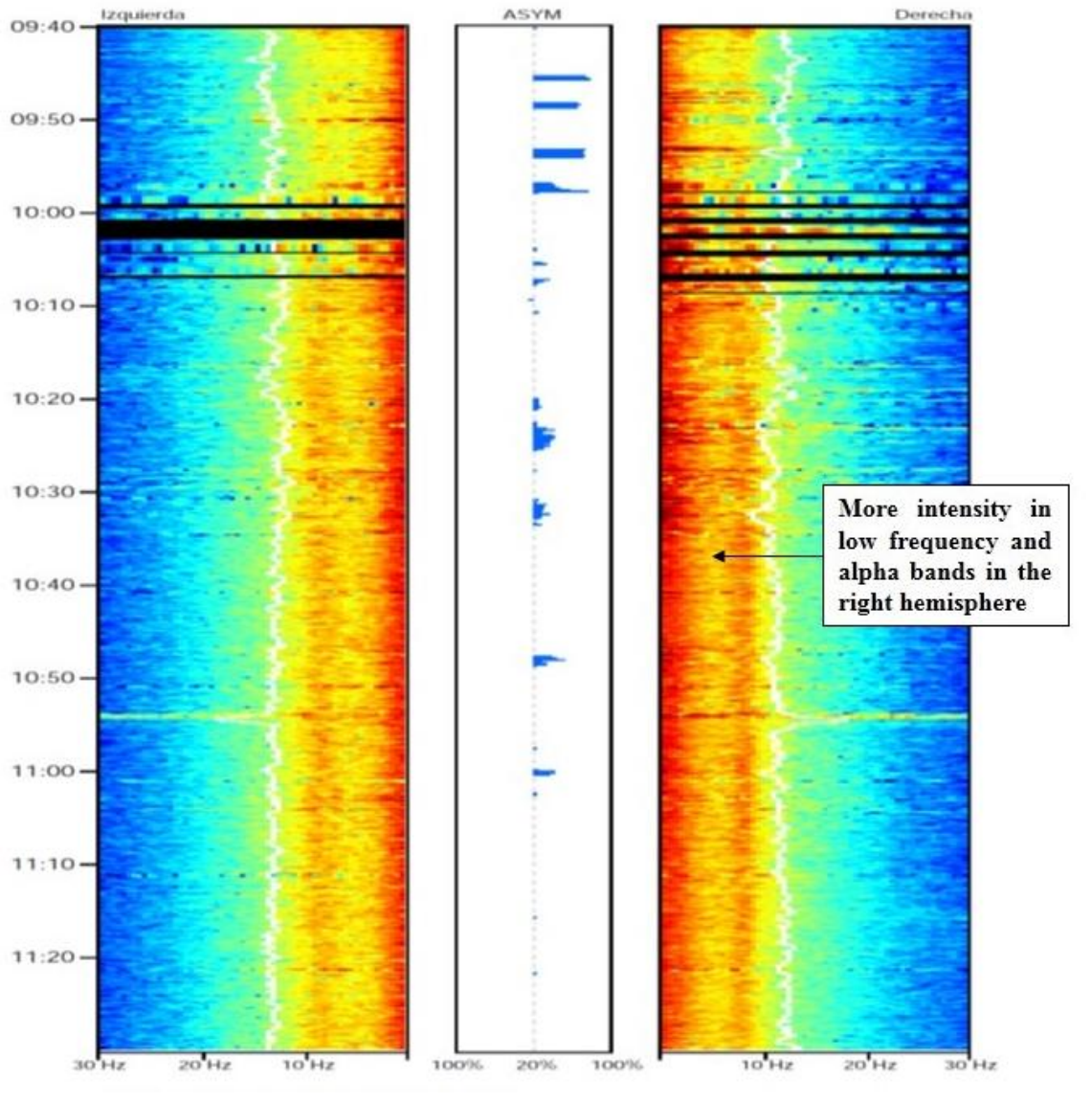

Fig 2. DSA before frontal disconection
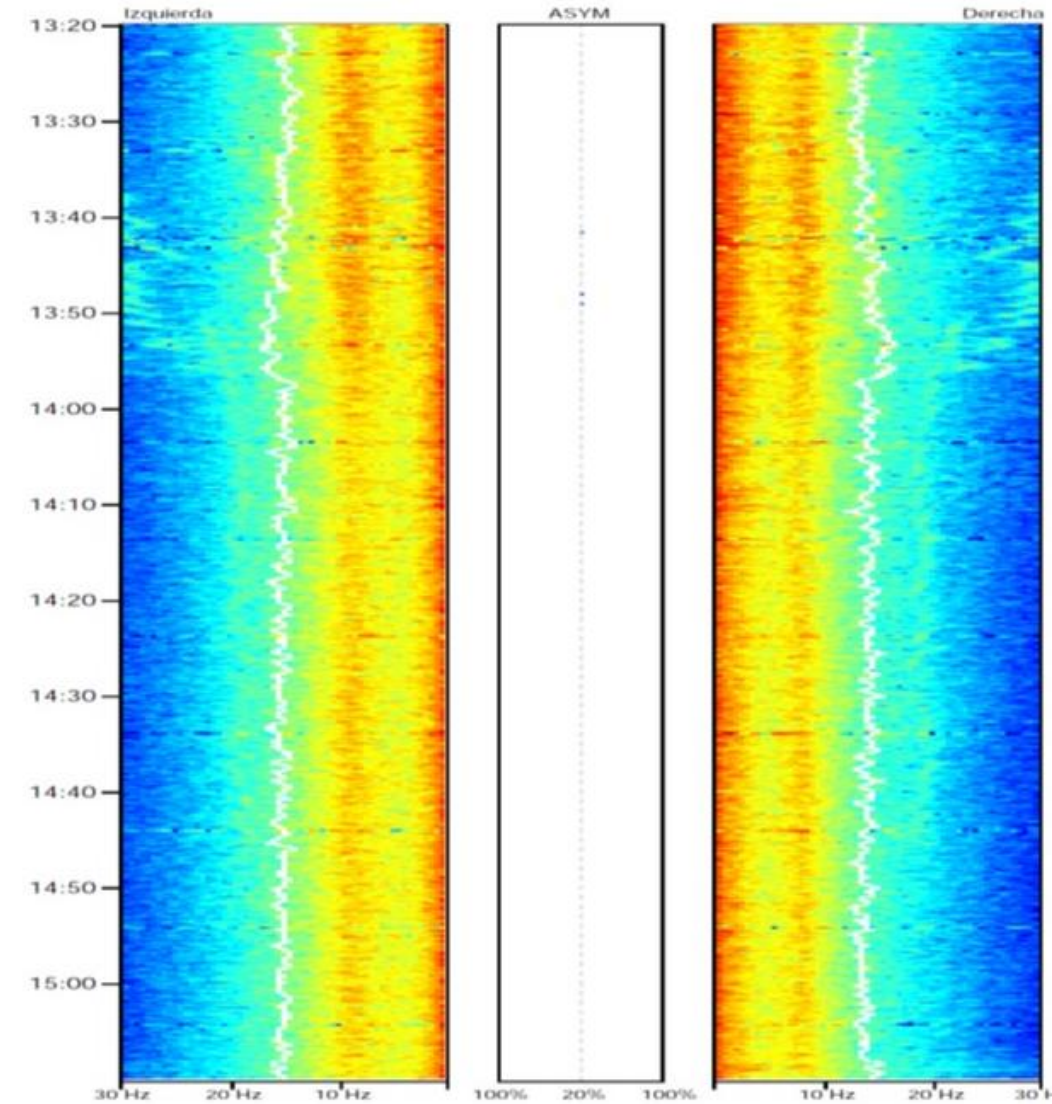

Fig 3. DSA during frontal disconection

\section{Discussion}

$\mathrm{FH}$ is a surgery rarely done in adults. There are some case reports about the use of Bilateral BIS in patients after FH, and they did not observe significant differences between the right and left-sided BIS values during the anesthesia. However, there are no publications about the use of DSA in FH. In this case, DSA displayed higher alpha power in the affected side (right) until the frontal disconnection. IDSA was useful for detecting differences between both hemispheres and monitoring the effectiveness of the surgery, because the epileptogenic focus was in the frontal area.This parameter allows the anesthesiologist to know that in future surgeries, the patient won't have differences in DSA between the two hemispheres.

\section{References:}

-Purdon PL, Sampson A, Pavone KJ, Brown EN. Clinical Electroencephalography for anesthesiologists. Anesthesiology, 2015, October;123(4):1-24.

- Kira S, Okuda K. Anesthetic management of a patient after functional hemispherectomy using bilateral bispectral index monitoring. M.E.J.Anesth. 2014;22(6):627-8. 